\title{
Composición florística y diversidad arbórea post quema de una formación subxerófila en el valle de Chanchamayo, Perú
}

\author{
Floristic composition and diversity of post-burn tree vegetation \\ of a subxerophilous formation in the Chanchamayo valley, Peru
}

Julio L. Collazos ${ }^{1, \star}$, Renzo Elejalde ${ }^{1}$, Norah J. Vega ${ }^{1}$ y Sonia Palacios-Ramos ${ }^{2}$

\begin{abstract}
Recibido: 04 febrero 2021 | Aceptado: 20 junio 2021 | Publicado en línea: 30 junio 2021 Citación: Collazos, JL; Elejalde, R; Vega, NJ; Palacios-Ramos, S. 2021. Composición florística y diversidad arbórea post quema de una formación subxerófila en el valle de Chanchamayo, Perú. Revista Forestal del Perú 36(1): 93-106. DOI: http://dx.doi.org/10.21704/rfp.vli36.1706
\end{abstract}

\begin{abstract}
Resumen
Los incendios forestales constituyen a nivel mundial una amenaza a la biodiversidad. En Perú, la tendencia de la pérdida de superficies boscosas va en aumento. En el valle de Chanchamayo (Junín, Perú), son frecuentes los incendios de origen antrópico con fines de cambio de uso de suelo para fines agropecuarios. La regeneración natural de estas áreas afectadas sigue siendo poco estudiada, por tanto, la información sobre su composición florística y diversidad siguen siendo escasas. En el presente estudio, se evalúa la composición florística y diversidad de la vegetación arbórea de una formación subxerófila en el valle de Chanchamayo, después de 20 meses de haber sido intervenida por quemas de origen antrópico. Se estableció un transecto de $2 \mathrm{~m} \times 500 \mathrm{~m}(0.1 \mathrm{ha})$; donde se registraron todos los individuos con DAP (medido a $1.3 \mathrm{~m}$ del suelo) $\geq 2.5 \mathrm{~cm}$. Se registraron 136 individuos, distribuidos en 45 especies y 29 familias. Las tres familias más abundantes fueron Lacistemataceae, Melastomataceae y Myrtaceae. La primera estuvo representada por una sola especie, que además fue la más abundante, Lacistema aggregatum (P.J. Bergius) Rusby, con 27 individuos, representando el $19.85 \%$ del total. Las siguientes más abundantes fueron Miconia ibaguensis (Bonpl.) Triana (10.29 \%) y Didymopanax morototoni (Aubl.) Decne. \& Planch. (5.88 \%). El transecto estudiado presenta similitudes florísticas con una formación subxerófila cercana y conservada, así como una diversidad $(\alpha=23.5)$ superior a esta y a otras formaciones vegetales de sucesión secundaria temprana del valle de Chanchamayo. Sin embargo, ante la ausencia de evaluaciones previas, la determinación de las repercusiones de la quema en el mismo, en términos de dinámica, diversidad y composición, dependerá de los monitoreos posteriores.
\end{abstract}

Palabras clave: regeneración natural, sucesión secundaria, incendios forestales, impacto antrópico

\footnotetext{
${ }^{1}$ Círculo de Investigación de Bosques Secos del Perú, Facultad de Ciencias Forestales, Universidad Nacional Agraria La Molina (UNALM), Av. La Molina s/n, La Molina, Lima, Perú.

${ }^{2}$ Facultad de Ciencias Forestales, Universidad Nacional Agraria La Molina (UNALM), Av. La Molina s/n, La Molina, Lima, Perú.

*Autor de Correspondencia: leonardo.collazos@gmail.com
} 


\begin{abstract}
Forest fires are a global threat to biodiversity. In Peru, the trend of forest loss is increasing. In the Chanchamayo valley (Junin, Peru), fires of anthropic origin with the purpose of changing land use for agriculture are frequent. The natural regeneration of these affected areas remains poorly studied, therefore, information on their floristic composition and diversity is still scarce. In the present study, the floristic composition and diversity of the arboreal vegetation of a subxerophilous formation in the Chanchamayo valley are evaluated, after 20 months of having been disturbed by burns of anthropogenic origin. A $2 \mathrm{~m} \times 500 \mathrm{~m}(0.1 \mathrm{ha})$ transect was installed; where all individuals with $\mathrm{DBH}$ (measured at $1.3 \mathrm{~m}$ from the ground) $\geq 2.5 \mathrm{~cm}$ were registered. 136 individuals were registered, distributed in 45 species and 29 families. The three most abundant families were Lacistemataceae, Melastomataceae and Myrtaceae. The first one was represented by a single species that, moreover, was the most abundant Lacistema aggregatum (P.J. Bergius) Rusby, with 27 individuals, equivalent to $19.85 \%$ of the total abundance. The next most abundant were Miconia ibaguensis (Bonpl.) Triana (10.29 \%) and Didymopanax morototoni (Aubl.) Decne. \& Planch. (5.88 \%). The evaluated transect presents floristic similarities with a nearby conserved subxerophilous formation and a diversity ( $\alpha=23.5)$ superior to this and to other early secondary succession plant formations of the Chanchamayo valley. Nevertheless, in absence of prior monitoring, determining the impact of burning on this plant formation in terms of dynamic, diversity and composition will depend on subsequent censuses.
\end{abstract}

Key words: natural regeneration, secondary succession, forest fires, anthropic impact

\section{Introducción}

Los incendios forestales son considerados una preocupación a nivel mundial por constituir una amenaza para la biodiversidad (Martínez et al. 2013). Se sabe que provocan daños a nivel de la biósfera y se les considera la tercera causa en la pérdida y degradación de grandes extensiones de terrenos forestales; además, son una fuente significativa de emisiones de gases de efecto invernadero (Martínez et al. 2013). Según la FAO (2020), a nivel mundial, la tasa de pérdida de bosques en la década 2010-2020 ha sido de 4.7 millones de hectáreas al año; si bien en Sudamérica la pérdida neta de superficie forestal ha disminuido con respecto a décadas anteriores, en Perú la tendencia ha sido inversa. La pérdida de superficie boscosa en el territorio peruano se encuentra en aumento desde el año 2001, alcanzando en el 2015 el máximo valor de las dos últimas décadas (MINAGRI 2016). Los incendios de origen antrópico con fines de cambio de uso de suelo para fines agropecuarios son frecuentes en nuestro país (Manta 2005), entre los años 2012 y 2016 se vieron afectadas aproximadamente
187600 hectáreas por dicho impacto (SERFOR 2018); en el departamento de Junín, los bosques del valle de Chanchamayo continúan siendo afectados por estas intervenciones (Palacios et al. 2018, Echía et al. 2019).

Las formaciones subxerófilas pueden ser consideradas transiciones entre bosques lluviosos y ecosistemas estacionalmente secos, cuya ubicación está determinada tanto por la estacionalidad de la precipitación como por las propiedades del suelo, temperatura, sequías, fuego y herbivoría (Palacios y Reynel 2011, Pennington et al. 2018). Asimismo, presentan especies vegetales con características diferenciadas, inusuales en bosques húmedos, que les brindan resistencia al fuego como cortezas gruesas y corchosas, ramas terminales gruesas y órganos subterráneos que pueden generar nuevos brotes (Palacios y Reynel 2011, Simon y Pennington 2012).

En el valle de Chanchamayo es posible encontrar estas formaciones embebidas en forma de pequeños fragmentos dentro de una gran matriz de vegetación típica de bosque húmedo premontano (Palacios et al. 2018). Su afinidad 
florística con bosques tropicales estacionalmente secos (BTES) de Brasil las ha incluido en el grupo florístico de BTES llamado Brasil Central (Banda et al. 2016).

La marcada diferencia en composición florística de las formaciones subxerófilas con otros bosques, en especial los estacionalmente secos; y la ausencia de áreas protegidas que contengan este tipo de vegetación amenazada por frecuentes quemas de origen antrópico, rocería y cambio de uso de suelo (Palacios y Reynel 2011, Palacios 2017, Palacios et al. 2018) hacen que el estudio de estas formaciones semisecas sea de suma importancia para la conservación. De no priorizarse ello, dentro del territorio nacional se podría perder este ecosistema con condiciones particulares y especies de distribución restringida (Banda et al. 2016). A esta problemática se suma el limitado conocimiento de la estructura de las formaciones subxerófilas antes de las quemas y los escasos estudios acerca de la regeneración natural de las mismas (Echía et al. 2019, Quintero Cardozo et al. 2020). Los cambios en la composición florística y reducción de la diversidad en este relicto constituyen una posible consecuencia de las perturbaciones causadas por quemas, que es necesario monitorear y analizar a mediano y largo plazo, así como el estudio del proceso de regeneración de la vegetación, con la finalidad de emplear esta nueva información para futuros esfuerzos de conservación.

En ese sentido, la presente investigación tiene como objetivo caracterizar la composición florística y diversidad de un relicto de bosque subxerófilo en el valle de Chanchamayo, Junín, Perú, luego de 20 meses de haber sido intervenido por quemas de origen antrópico.

\section{Materiales y métodos}

\section{Área de estudio}

La zona de estudio se encuentra en Perú, específicamente en una formación subxerófila en el valle del río Chanchamayo, en el departamento de Junín, provincia Chanchamayo y distrito San Ramón, entre las coordenadas UTM 18L 460000-480000 E y 878000-8800000 N (Figu- ra 1). El valle se ubica al noroeste de la intrusión San Ramón, en el flanco oriental de la cordillera de Huaytapallana y es parte de la cuenca del río Perené; el río Chanchamayo surge de la confluencia de los ríos Oxabamba y Tulumayo a la altura de la ciudad de San Ramón hasta que se une al río Paucartambo (Palacios 2017).

El clima del valle, según la clasificación de Thornthwaite, es cálido lluvioso (SENAMHI 2018). Posee una estación lluviosa (diciembre a mayo) y una seca (junio a agosto), con una precipitación total anual promedio de entre 1970 a $2100 \mathrm{~mm}$; la temperatura es elevada, con una media anual de $23.1^{\circ} \mathrm{C}$ y una desviación de hasta $7{ }^{\circ} \mathrm{C}$ en los meses más cálidos o más fríos (SENAMHI 2018).

La vegetación es predominantemente secundaria; mientras que las zonas sin intervenir son clasificadas como "bosque basimontano de yungas” (MINAM 2019). Estos se caracterizan por presentar tres estratos diferenciados y dosel cerrado de entre 25 y 35 metros (MINAM 2019); en donde es frecuente encontrar individuos leñosos de las familias Euphorbiaceae, Fabaceae, Lauraceae, Melastomataceae, Moraceae y Rubiaceae (Antón y Reynel 2004, Marcelo-Peña y Reynel 2014, Armey 2019). En cuanto a las formaciones subxerófilas dispersas presentes, es posible encontrar en ellas especies que no se hallan en los bosques húmedos del valle, como Astronium fraxinifolium Schott, Curatella americana L., Machaerium pilosum Benth., Roupala montana Aubl., entre otras; las cuales son propias de BTES o sabanas (Palacios et al. 2018).

El área de estudio tiene una historia de intervenciones que incluyen quemas, dos de las últimas fueron registradas en 2005 y 2009 (Palacios y Reynel 2011). Luego del establecimiento de una parcela permanente de muestreo en el año 2009 (P-GSX) (Palacios 2017), con coordenadas UTM 18L 462779E y $8772597 \mathrm{~N}$, se conservó el área sin quemas, permitiendo la recuperación de la cobertura vegetal; sin embargo, en el año 2018 se realizó una nueva intervención en las cercanías de la parcela, sin afectar el interior de esta, con el fin de ampliar el espacio agrícola (Palacios et al. 2018). Luego de 20 meses de dicho impacto, se realizó el pre- 


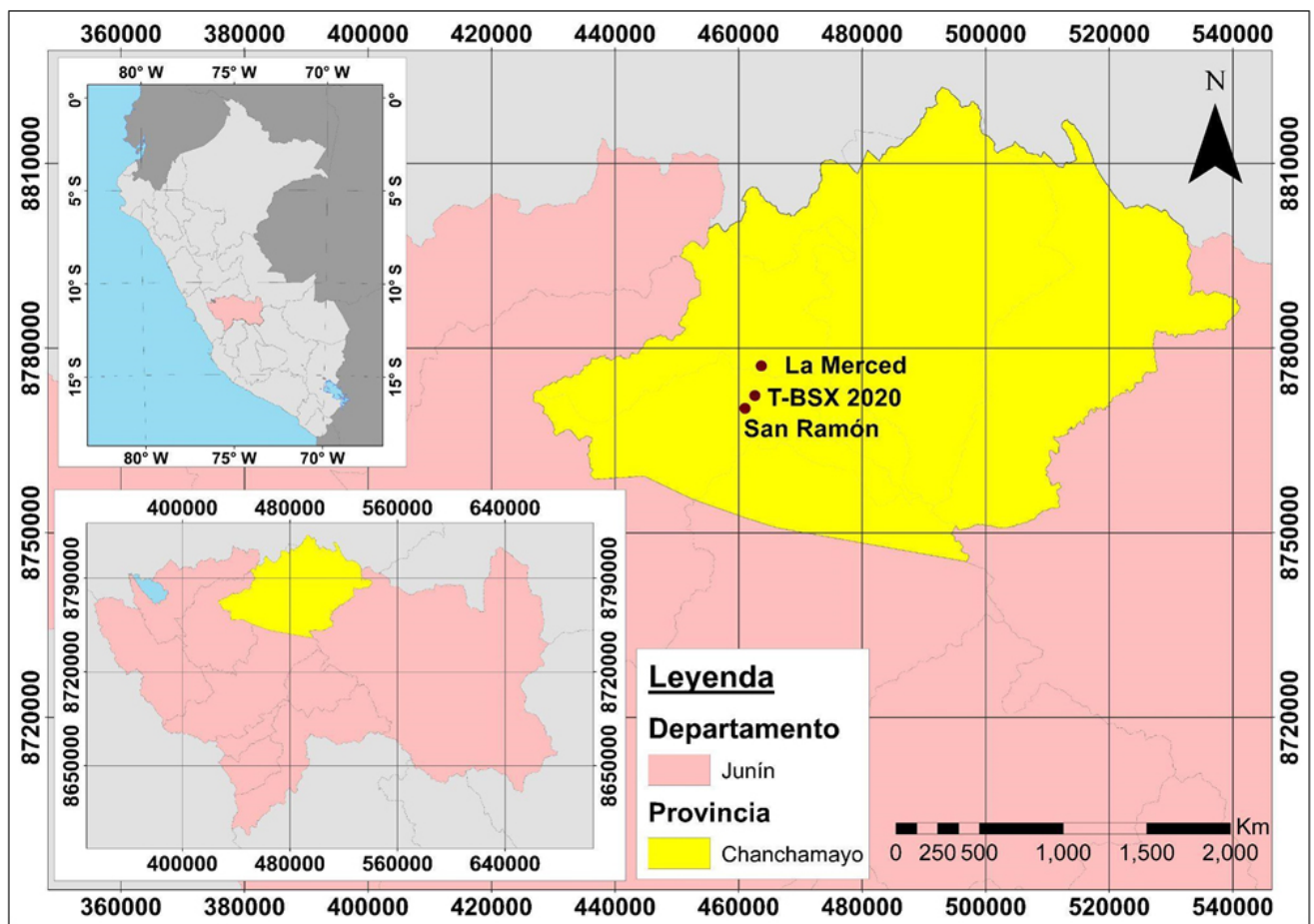

Figura 1. Mapa de ubicación del transecto T-BSX 2020, en el departamento de Junín, Perú.

sente estudio en esta zona intervenida a aproximadamente 400 metros de la P-GSX.

\section{Establecimiento del transecto de muestreo y colecta botánica}

Siguiendo la metodología propuesta por Gentry y Ortiz (1993), se estableció un transecto (T-BSX 2020) de $500 \mathrm{~m} \times 2 \mathrm{~m}$ en enero del 2020, cuyas coordenadas son: UTM 18L $462652 \mathrm{E} 8772206 \mathrm{~N}$. Se registraron todos los individuos con diámetro a la altura del pecho (DAP) mayor o igual a $2.5 \mathrm{~cm}$, tomando de referencia estudios previos en el valle de Chanchamayo (Cáceres 2005, Palacios 2017, Echía et al. 2019, Quintero 2019); y el taxón en la medida que fue posible en campo. Se colectaron muestras botánicas de todos los individuos evaluados.

\section{Identificación botánica}

La identificación botánica se realizó en cuatro etapas: (i) se consultó a especialistas del
Herbario de la Facultad de Ciencias Forestales de la UNALM; (ii) se utilizaron dos claves de identificación: "A field guide to the families and genera of woody plants of northwest South America: (Colombia, Ecuador, Perú): with supplementary notes" (Gentry 1996) y "Clave para identificar grupos de familias de Gymnospermae y Angiospermae del Perú" (Vásquez y Rojas 2016); (iii) se contrastaron las muestras con los especímenes depositados en el Herbario de la Facultad de Ciencias Forestales y (iv) se verificó la nomenclatura correcta de los individuos con la ayuda del "Catálogo de los árboles del Perú” (Vásquez et al. 2018) y la base de datos IPNI (IPNI 2020). Posteriormente, las muestras fueron depositadas en el Herbario de la Facultad de Ciencias Forestales UNALM (MOL).

\section{Análisis de los datos}

Se determinó la riqueza y abundancia de la zona estudiada mediante el conteo del número de individuos, especies, géneros y familias 


\begin{tabular}{|c|c|c|}
\hline Familia & Especie & Abundancia \\
\hline Anacardiaceae & Mauria heterophylla Kunth & 4 \\
\hline Araliaceae & Didymopanax morototoni Decne. \& Planch. & 8 \\
\hline Asteraceae & Asteraceae Indet. 1 & 1 \\
\hline Asteraceae & Asteraceae Indet. 2 & 1 \\
\hline Chrysobalanaceae & Hirtella burchellii Britton & 5 \\
\hline Chrysobalanaceae & Hirtella sp. & 1 \\
\hline Clusiaceae & Clusia hammeliana Pipoly & 1 \\
\hline Combretaceae & Terminalia amazonia (J.F. Gmel.) Exell & 2 \\
\hline Erythroxylaceae & Erythroxylum mucronatum Benth & 4 \\
\hline Euphorbiaceae & Maprounea guianensis Aubl. & 2 \\
\hline Euphorbiaceae & Sapium glandulosum (L.) Morong & 6 \\
\hline Fabaceae & Machaerium hirtum (Vell.) Stellfeld & 5 \\
\hline Fabaceae & Machaerium sp. & 2 \\
\hline Fabaceae & Tachigali peruviana (Dwyer) Zarucchi \& Herend. & 1 \\
\hline Hypericaceae & Vismia cf. macrophylla Kunth & 1 \\
\hline Lacistemataceae & Lacistema aggregatum (P.J.Bergius) Rusby & 27 \\
\hline Lauraceae & Persea caerulea (Ruiz \& Pav.) Mez & 3 \\
\hline Lythraceae & Physocalymma scaberrimum Pohl & 5 \\
\hline Malpighiaceae & Byrsonima spicata Poepp. ex Spreng. & 3 \\
\hline Malpighiaceae & Heteropterys laurifolia A.Juss. & 3 \\
\hline Malvaceae & Ceiba insignis (Kunth) P.E.Gibbs \& Semir & 1 \\
\hline Malvaceae & Ceiba sp. & 1 \\
\hline Malvaceae & Guazuma ulmifolia Lam. & 2 \\
\hline Malvaceae & Malvaceae Indet. & 1 \\
\hline Melastomataceae & Miconia ibaguensis Schltdl. & 14 \\
\hline Meliaceae & Cedrela fissilis Vell. & 1 \\
\hline Myrsinaceae & Myrsine coriacea (Sw.) R.Br. & 2 \\
\hline Myrtaceae & Psidium guineense $\mathrm{Sw}$. & 4 \\
\hline Myrtaceae & Myrtaceae Indet. 1 & 5 \\
\hline Myrtaceae & Myrtaceae Indet. 2 & 1 \\
\hline Nyctaginaceae & Neea sp. & 1 \\
\hline Piperaceae & Piper aduncum L. & 1 \\
\hline
\end{tabular}

Cuadro 1. Composición florística del T-BSX 2020. 


\begin{tabular}{|l|l|c|}
\hline \multicolumn{1}{|c|}{ Familia } & \multicolumn{1}{c|}{ Especie } & Abundancia \\
\hline Piperaceae & Piper hispidum Sw. & 2 \\
\hline Polygonaceae & Coccoloba sp. 1 & 1 \\
\hline Polygonaceae & Coccoloba sp. 2 & 1 \\
\hline Proteaceae & Roupala montana Aubl. & 1 \\
\hline Rutaceae & Dictyoloma peruvianum Planch. & 1 \\
\hline Salicaceae & Banara guianensis Aubl. & 1 \\
\hline Salicaceae & Casearia sp. 1 & 1 \\
\hline Salicaceae & Casearia sp. 2 & 1 \\
\hline Sapindaceae & Allophylus floribundus Radlk. & 1 \\
\hline Sapindaceae & Allophylus leiophloeus Radlk. & 3 \\
\hline Urticaceae & Cecropia sp. & 1 \\
\hline Verbenaceae & Duranta sp. & 136 \\
\hline Indeterminada & Indeterminada & 136 \\
\hline Total & & \\
\hline
\end{tabular}

Cuadro 1 (continuación). Composición florística del T-BSX 2020.

encontradas en el transecto; asimismo, se calculó la abundancia relativa de estos. Además, se revisó la distribución de las especies utilizando la base de datos de DRYFLOR (DRYFLOR 2020). Finalmente, se comparó la afinidad del área de estudio con otras dentro del valle de Chanchamayo a través de un dendrograma de agrupamiento, mediante el índice de disimilaridad de Bray-Curtis y el método de distancia de medias aritméticas no ponderadas (UPGMA), elaborado en el software de programación $\mathrm{R}$ 4.0.2 (R Core Team 2020) con el paquete "vegan" (Oksanen et al. 2019).

Se calculó el índice de diversidad alfa de Fisher (Fisher et al. 1943) mediante el programa PAST versión 2.17c (Hammer et al. 2001) y para el cociente de mezcla, que estima la heterogeneidad de la composición florística, se utilizó Excel 2016. Las fórmulas empleadas se presentan a continuación:

Índice de diversidad alfa de Fisher $(\alpha)$ (Fisher et al. 1943), $S=\alpha \cdot \ln (1+\mathrm{N} / \alpha)$, donde: $\mathrm{S}$ es el número de especies y $\mathrm{N}$ es el número de individuos.
Cociente de mezcla (C.M.), C.M. = S/N, donde: $\mathrm{S}$ es el número de especies y $\mathrm{N}$ es el número de individuos.

\section{Resultados}

Se hallaron 136 individuos, distribuidos en 45 especies y en 29 familias (Cuadro 1). De estas 29 familias botánicas registradas, 18 contienen más del $90 \%$ de los individuos presentes. La más abundante fue Lacistemataceae, equivalente al $19.85 \%$ del total de individuos encontrados en el transecto; sucedida por Melastomataceae (10 \%), Myrtaceae (7 $\%)$, Araliaceae (6 \%), Fabaceae (6 \%) y Euphorbiaceae (6\%); seguidas por familias que representan menos del $5 \%$ del total en cada caso (Figura 2). Se registró un elevado porcentaje de familias monoespecíficas (Cuadro 2). Por otro lado, Malvaceae fue la familia con mayor cantidad de especies (4), seguida por Fabaceae, Myrtaceae y Salicaceae, con 3 especies en cada una de ellas. En cuanto a la familia Myrtaceae, 6 individuos no pudieron 


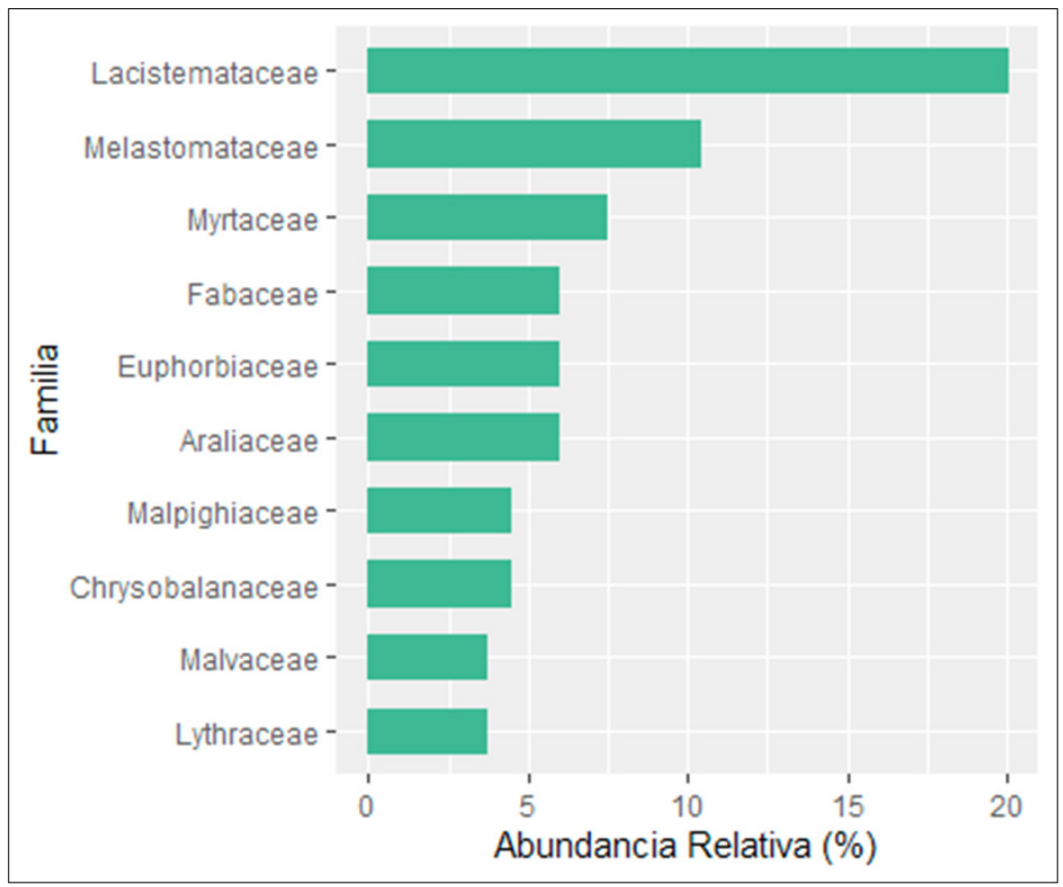

Figura 2. Abundancia relativa por familias del transecto T-BSX 2020, en el departamento de Junín, Perú.

ser identificados a nivel de especie, pero sí se determinaron 3 morfotipos.

Dado que la mayoría de los géneros son monoespecíficos no se ha considerado un análisis a nivel de género. A nivel de especie, como se observa en la Figura 3, Lacistema aggregatum (P.J.Bergius) Rusby, con 27 individuos (19.85\%), es la más abundante, seguida de Miconia ibaguensis (Bonpl.) Triana, con 14 individuos (10.29 \%) y Didymopanax morototoni (Aubl.) Decne. \& Planch. con 8 individuos $(5.88 \%)$. Las tres especies más abundantes fueron las únicas representantes de sus respectivas familias (Cuadro 1).

En la Figura 4, en una comparación entre transectos establecidos en bosques secundarios del valle de Chanchamayo, se aprecia la cercanía en composición florística entre el transecto de la presente investigación y un transecto de vegetación subxerófila (T-GSX), ubicado al interior de la parcela P-GSX, establecido en un estudio previo (Palacios 2017). A su vez, es- tos dos transectos se encuentran más próximos florísticamente al transecto evaluado a 15 años posteriores a una intervención antropogénica sin quemas (T-Q15) (Quintero 2019) que a los transectos evaluados a 10 y 25 años posteriores a quemas (T-EE10 y T-EE25, respectivamente) (Echía et al. 2019).

Los cálculos de diversidad en el transecto T-BSX 2020 muestran el valor de alfa de Fisher (a) (23.5) más alto comparado con otros estudios del valle de Chanchamayo (Cáceres 2005, Palacios 2017, Echía et al. 2019, Quintero 2019, Quintero Cardozo et al. 2020). Se obtuvo un coeficiente de mezcla de 1:3 (Cuadro 3).

\section{Discusión}

La composición de familias del área de estudio presenta altas coincidencias $(75.9 \%)$ con las reportadas por Echía et al. (2019) en transectos de bosques secundarios regenerados tras quemas y con las reportadas por Quintero (2019) en transectos de bosques secundarios 
Composición florística y diversidad arbórea post quema de una formación subxerófila en el valle de Chanchamayo, Perú

\begin{tabular}{|l|c|c|}
\hline Cantidad de especies & $\mathbf{N}^{\circ}$ de familias & Abundancia Relativa (\%) \\
\hline Con 4 especies & 1 & 3.45 \\
\hline Con 3 especies & 3 & 10.34 \\
\hline Con 2 especies & 7 & 24.14 \\
\hline Con 1 especie & 18 & 62.07 \\
\hline
\end{tabular}

Cuadro 2. Especies por familia en el T-BSX 2020.

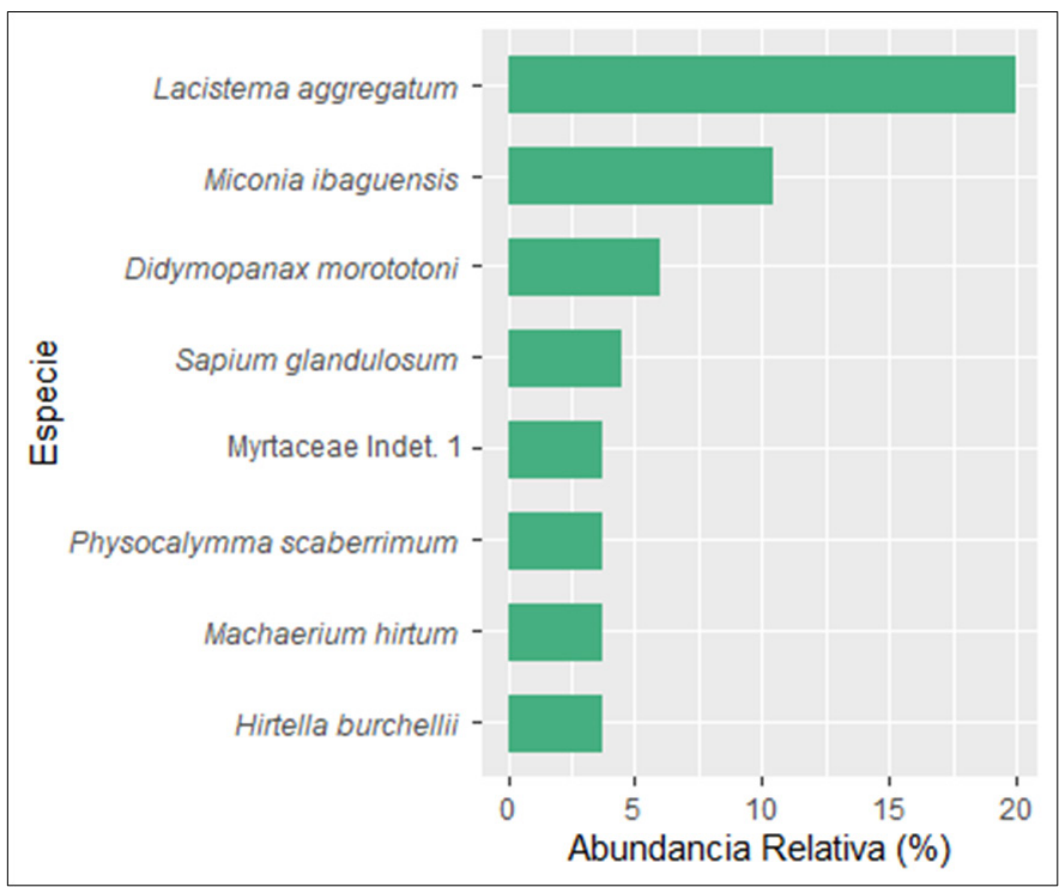

Figura 3. Abundancia relativa por especie del transecto T-BSX 2020, en el departamento de Junín, Perú.

regenerados tras intervención antrópica sin quemas. Destacan como familias compartidas Fabaceae, Piperaceae, Malvaceae, Urticaceae, Euphorbiaceae, Myrtaceae y Anacardiaceae; sin embargo, mientras en los estudios mencionados, en estadíos tempranos, son más comunes los individuos de las familias Fabaceae, Piperaceae y Urticaceae (Echía et al. 2019, Quintero 2019), en la zona de estudio, son más frecuentes los individuos de las familias Lacistemataceae, Melastomataceae y Myrtaceae. Asimismo, comparando bosques pioneros en- tre los estudios citados, las familias que solo se encontraron en el transecto subxerófilo T-BSX 2020 fueron Araliaceae, Chrysobalanaceae, Hypericaceae, Lythraceae, Myrsinaceae y Rutaceae. Por otro lado, Araliaceae, Hypericaceae y Rutaceae se registraron también en los bosques de 20 a 50 años de regeneración posteriores a disturbios (Quintero 2019).

El elevado porcentaje de familias monoespecíficas es un aspecto común de los bosques montanos y premontanos del valle del río Chanchamayo (Antón y Reynel 2004, Palacios 
y Reynel 2011, Palacios 2017, Echía et al. 2019, Quintero 2019). En cuanto a bosques impactados por quemas, esta característica se repite solo en los bosques secundarios de cinco años de regeneración postfuego, mientras que en los bosques de mayor tiempo post intervención por quemas (10 y 25 años) la proporción se hace más equitativa (Echía et al. 2019). En el transecto T-BSX 2020, Malvaceae, una familia pantropical, fue la única que presentó cuatro especies (Cuadro 2), entre ellas Guazuma ulmifolia Lam., de amplia distribución y reportada en zonas de bosques regeneradas desde quemas (Middleton et al. 1997, Echía et al. 2019).

También destaca Lacistema aggregatum por ser la más abundante (Figura 3); esto puede atribuirse a que se trata de una especie heliófita durable (Quintero 2019), habitualmente presente en claros de bosque, como los que aparecieron luego de la quema, además de su carácter cosmopolita; esta especie ha sido ampliamente registrada en ecosistemas tanto húmedos como estacionalmente secos del Neotrópico (Macbride 1941, Burger 1977, Lang y Knight 1983, Banda et al. 2016, Palacios 2017). Asimismo, las especies de la familia Melastomataceae son abundantes en bosques pioneros, en especial en áreas intervenidas (Antón y Reynel 2004,
Marcelo-Peña y Reynel 2014, Palacios 2017, Armey 2019, Echía et al. 2019). Esto puede explicarse por la respuesta fotoblástica positiva de sus semillas, favorable para su germinación en claros de bosque, así como por el pequeño tamaño de estas, que les permite tener una mayor longevidad y evitar la depredación (Silveira et al. 2013). En cuanto a M. ibaguensis, si bien no se ha registrado en los BTES (DRYFLOR 2020) ni en otros bosques del valle de Chanchamayo, múltiples individuos del género Miconia no llegan a ser identificados hasta nivel de especie (Antón y Reynel 2004, Palacios 2017, Armey 2019), lo que dificulta dilucidar su distribución en el valle y realizar comparaciones entre estudios del área. Por otro lado, Didymopanax morototoni, se ha registrado en bosques semideciduos estacionales del Cerrado; en el bosque húmedo amazónico con estación seca del Acre y en las formaciones vegetales subxerofíticas de Colombia, tanto en áreas regeneradas después de quemas como en intactas y transicionales (Moscovich et al. 2010, Xavier et al. 2011, Numata et al. 2017, Pereira et al. 2017, Sardi et al. 2018).

En el transecto T-GSX (Palacios 2017), ubicado al interior de la parcela de vegetación subxerófila P-GSX, se obtuvo una coincidencia del

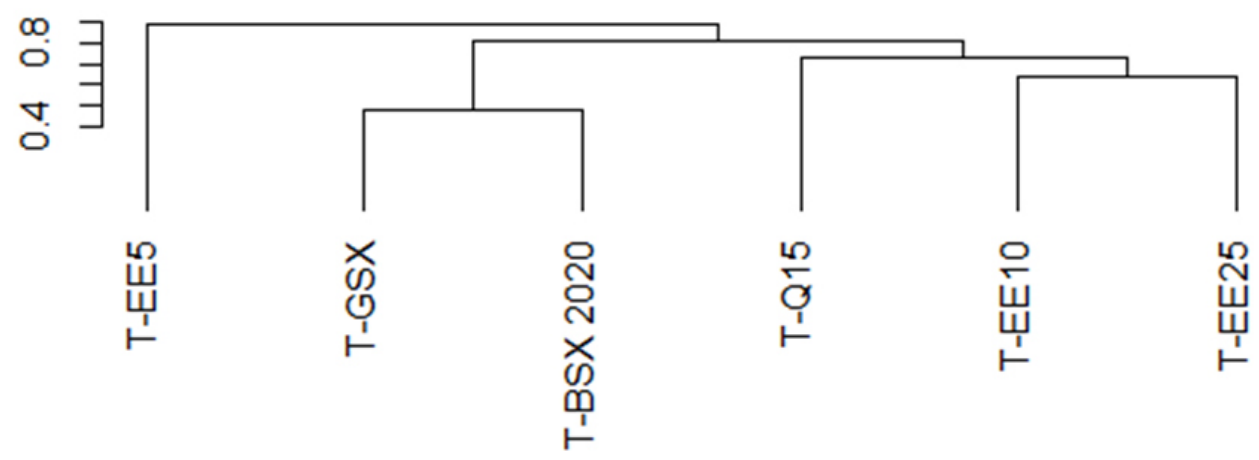

Figura 4. Dendrograma de agrupamiento entre la composición florística de otros estudios en el valle de Chanchamayo, en el departamento de Junín, Perú. Las siglas indican los siguientes transectos: T-BSX 2020, el transecto del presente estudio; T-GSX, transecto establecido dentro de una parcela con vegetación subxerófila (Palacios 2017); T-EE5, T-EE10, T-EE25, transectos de cinco, diez y veinticinco años de regeneración posteriores a quemas, respectivamente (Echía et al. 2019); T-Q15, transecto de 15 años de regeneración posteriores a intervenciones sin quemas (Quintero 2019). 


\begin{tabular}{|c|c|c|c|}
\hline Transecto & Tipo de vegetación & $\begin{array}{l}\text { Cociente de } \\
\text { mezcla }\end{array}$ & $\begin{array}{l}\text { Alfa de Fisher } \\
\qquad(\alpha)\end{array}$ \\
\hline T-BSX 2020 & Subxerófila tras quemas & 0.33 & 23.50 \\
\hline T-Q15 (Quintero 2019) & Húmeda tras intervención sin quemas & 0.21 & 20.22 \\
\hline T-EE25 (Echía et al. 2019) & Húmeda tras quemas & 0.10 & 20.20 \\
\hline $\begin{array}{l}\text { T-Q20 (Quintero Cardozo et } \\
\text { al. 2020) }\end{array}$ & Húmeda tras intervención sin quemas & 0.07 & 17.9 \\
\hline T-C15 (Cáceres 2005) & Húmeda tras intervención sin quemas & 0.51 & 16.08 \\
\hline T-GSX (Palacios 2017) & Subxerófila no intervenida & 0.16 & 14.92 \\
\hline T-C5 (Cáceres 2005) & Húmeda tras intervención sin quemas & 0.45 & 11.33 \\
\hline T-C10 (Cáceres 2005) & Húmeda tras intervención sin quemas & 0.33 & 10.49 \\
\hline $\begin{array}{l}\text { T-Q10 (Quintero Cardozo et } \\
\text { al. 2020) }\end{array}$ & Húmeda tras intervención sin quemas & 0.26 & 10.20 \\
\hline T-EE5 (Echía et al. 2019) & Húmeda tras quemas & 0.17 & 5.23 \\
\hline T-EE10 (Echía et al. 2019) & Húmeda tras quemas & 0.13 & 3.86 \\
\hline
\end{tabular}

Cuadro 3. Comparación de la diversidad del T-BSX 2020 con otros estudios.

$40 \%$ con las especies del presente estudio, lo que refleja una mayor cercanía florística entre ambos en relación a los otros sitios (Figura 4). A pesar de que el transecto del área conservada presenta un número significativamente mayor de individuos, sólo presenta una especie más que el transecto T-BSX 2020. Todas las especies reportadas en este, a excepción de Erythroxylum mucronatum Benth. y $M$. ibaguensis, se encuentran registradas en colectas de otros ecosistemas estacionalmente secos neotropicales (DRYFLOR 2020), principalmente del grupo florístico Brasil Central, asociados al Cerrado brasileño. Esto coincide con la propuesta de afinidad florística de los bosques subxerófilos del área de estudio con estos BTES (Palacios y Reynel 2011, Banda et al. 2016).

Respecto a la diversidad, comparándose con otras formaciones vegetales del valle (Cuadro $3)$, se obtuvo un valor de diversidad alfa de Fisher (23.5) superior al registrado en el transecto (T-GSX) establecido en la zona conservada del área de estudio (P-GSX); al de bosques secundarios intervenidos por quemas; y de bosques secundarios intervenidos por rocerías sin quemas (Antón y Reynel 2004, Cáceres 2005, Echía 2013, Palacios 2017, Quintero Cardozo et al. 2020). Esto podría estar relacionado con las estrategias adaptativas de las especies. En estudios postfuego realizados en BTES neotropicales se menciona que estos bosques son menos vulnerables al fuego en comparación con los bosques húmedos debido a que las especies de flora presentan adaptaciones a esta perturbación, como rebrotes, baja mortalidad y rápido reclutamiento gracias a estrategias de dispersión, incluso pudiendo alcanzar índices de diversidad comparables a bosques secundarios sin disturbios o de estadíos sucesionales más avanzados (Kennard et al. 2002, Otterstrom et al. 2006, Simon y Pennington 2012, Gutiérrez Rincón 2019). Por otro lado, por cada tres individuos registrados, es posible encontrar una especie diferente (C.M.:1:3), un resultado que se encuentra dentro de la tendencia general para los estadíos sucesionales tempranos del valle de Chanchamayo (Antón y Reynel 2004, Quintero 2019), lo que indica una elevada heterogeneidad en la composición florística.

La escasa evaluación cuantitativa previa a la quema dificulta determinar el efecto del fuego 
sobre la composición de la vegetación previamente existente. No obstante, la información disponible sobre la composición florística y diversidad del transecto establecido en el área cercana (Palacios 2017) permite un acercamiento para el análisis comparativo. Se ha señalado que las quemas ocurridas en 2005 y 2009 sobre el área de estudio afectaron la cobertura vegetal del sotobosque y el follaje de los árboles (Palacios 2017), semejante a lo ocurrido en la última intervención del 2018 en el área del transecto T-BSX 2020. Los resultados muestran una composición florística y valores de diversidad similares a los registrados en la zona conservada del área de estudio.

\section{Consideraciones finales}

La falta de estudios cuantitativos de la inusual composición florística y diversidad del área de estudio antes de la intervención, dificultan la determinación del efecto del fuego en el sitio. Si bien estos indicadores son similares a los presentados en una formación subxerófila conservada, es necesario el monitoreo en el mediano y largo plazo para poder determinar con certeza dicho efecto. En ese sentido, se sugiere conservar esta formación vegetal para dichos fines.

\section{Agradecimientos}

Los autores agradecemos a los Vicerrectorados de Investigación y Académico de la Universidad Nacional Agraria La Molina (UNALM) por brindarnos el financiamiento para la investigación; a Robin Fernandez Hilario, Italo Revilla, Gianlucca Monteverde y a Aniceto Daza del Herbario de la Facultad de Ciencias Forestales (MOL) de la UNALM, por contribuir con la identificación de las especies; a Eduardo Chu, administrador del IRD-Selva "Fundo Génova" de la UNALM, por su apoyo durante la fase de campo; a Pierina Tuesta, Myshell Catalán y Gino Rivera, por contribuir en el trabajo de colecta botánica, y finalmente al Círculo de Investigación de Bosques Secos del Perú (CIBOSEC).

\section{Referencias}

Antón, D; Reynel, C. (eds.). 2004. Relictos de bosques de excepcional diversidad en los Andes Centrales del Perú. Lima, Perú, UNALM. $323 \mathrm{p}$.

Armey, R. 2019. Diversidad arbórea en tres estadios sucesionales en bosques en la Selva Central del Perú. Tesis Ing. Lima, Perú, UNALM, $146 \mathrm{p}$.

Banda, RK; Delgado-Salinas, A; Dexter, K; Linares-Palomino, R; Oliveira-Filho, A; Prado, D; Pullan, M; Quintana, C; Riina, R; Rodriguez M., G; Weintritt, J; Acevedo-Rodriguez, P; Adarve, J; Alvarez, E; Aranguren B., A; Arteaga, J; Aymard, G; Castano, A; Ceballos-Mago, N; Cogollo, A; Cuadros, H; Delgado, F; Devia, W; Duenas, H; Fajardo, L; Fernandez, A; Fernandez, M; Franklin, J; Freid, E; Galetti, L; Gonto, R; Gonzalez-M., R; Graveson, R; Helmer, E; Idarraga, A; Lopez, $\mathrm{R}$; Marcano-Vega, $\mathrm{H}$; Martinez, O; Maturo, H; McDonald, M; McLaren, K; Melo, O; Mijares, F; Mogni, V; Molina, D; Moreno, N; Nassar, J; Neves, D; Oakley, L; Oatham, M; Olvera-Luna, A; Pezzini, F; Dominguez, O; Rios, M; Rivera, O; Rodriguez, N; Rojas, A; Sarkinen, T; Sanchez, R; Smith, M; Vargas, C; Villanueva, B; Pennington, RT. 2016. Plant diversity patterns in neotropical dry forests and their conservation implications. Science 353(6306):1383-1387. DOI: https:// doi.org/10.1126/science.aaf5080.

Burger, W (ed.). 1977. Flora costaricensis. Chicago, Estados Unidos de América, Field Museum of Natural History. 291 p. (Botanical Series, v. 40).

Cáceres, B. 2005. Diversidad de la composición florística de la microcuenca de Santa Rosa, Chanchamayo, Junín. Tesis Mg. Sc. Lima, Perú, UNALM. 241 p.

DRYFLOR (Red Florística Latinoamericana del Bosque Tropical Estacionalmente Seco). 2020. Latin American Seasonally Dry Tropical Forest Floristic Network website (en línea, base de datos). Consultado 20 dic. 2020. Disponible en: http://www.dryflor.info/data.

Echía, E. 2013. Composición y diversidad de la flora leñosa en bosques secundarios generados 
a partir de quemas en el valle de Chanchamayo, Junín. Tesis Ing. Lima, Perú, UNALM. 144 p.

Echía, E; Reynel, C; Manta, M. 2019. La flora leñosa establecida luego de las quemas en el valle de Chanchamayo-Selva central del Perú. Revista Forestal del Perú 34(1):83-101.

FAO (Organización de las Naciones Unidas para la Alimentación y la Agricultura, Italia). 2020. Evaluación de los recursos forestales mundiales 2020: principales resultados (en línea). Roma, Italia. 16 p. Consultado 27 dic. 2020. Disponible en http://www.fao.org/3/ CA8753ES/CA8753ES.pdf.

Fisher, RA; Corbet, AS; Williams, CB. 1943. The relation between the number of species and the number of individuals in a random sample of an animal population. The Journal of Animal Ecology 12(1):42-58.

Gentry, A. 1996. A field guide to the families and genera of woody plants of northwest South America (Colombia, Ecuador, Peru) with supplementary notes. Chicago, Estados Unidos de América, University of Chicago Press. 918 p.

Gentry, A; Ortiz, R. 1993. Patrones de composición florística en la Amazonía Peruana. In Kalliola, R; Puhakka, M; Danjoy, W (eds.). Amazonía Peruana: vegetación húmeda tropical en el llano subandino.Tuku, Finlandia, PAUT. p. 155-166.

Gutiérrez Rincón, AS. 2019. Efecto del fuego sobre el reclutamiento de especies leñosas en un bosque seco de la cuenca alta del Río Magdalena. Tesis Mg. Sc. Bogotá, Colombia, Universidad Distrital Francisco José de Caldas. 64 p.

Hammer, Ø; Harper, DA; Ryan, PD. 2001. PAST: Paleontological statistics software package for education and data analysis (en línea). Palaeontologia Electronica 4(1):1-9. Consultado 27 dic. 2020. Disponible en http://palaeo-electronica. org/2001 1/past/issue1 01.htm.

IPNI (International Plant Names Index). 2020. International plant names index. Kew, Reino Unido, The Royal Botanic Gardens, Consultado 28 dic. 2020. Disponible en http://www.ipni. org.
Kennard, DK; Gould, K; Putz, FE; Fredericksen, TS; Morales, F. 2002. Effect of disturbance intensity on regeneration mechanisms in a tropical dry forest. Forest ecology and management 162(2-3):197-208.

Lang, G; Knight, D. 1983. Tree growth, mortality, recruitment, and canopy gap formation during a 10 -year period in a tropical moist forest. Ecology 64(5):1075-1080. DOI: https://doi. org/10.2307/1937816.

Macbride, F. 1941.Flora of Peru: Elatinaceae. Chicago, Estados Unidos de América, Field Museum of Natural History. 566 p. (Botanical Series, v.13 pt. 4 n. $\left.{ }^{\circ} 1\right)$.

Manta, M. 2005. Evaluación de los incendios forestales en la provincia de Satipo, departamento de Junín, Perú. Lima, Perú, s.e. 59 p.

Marcelo-Peña, JL; Reynel, C. 2014. Patrones de diversidad y composición florística de parcelas de evaluación permanente en la selva central de Perú. Rodriguésia 65(1):35-47.

Martínez, G; Hernández, M; Ordoñez, J; Camacho, J. 2013. Régimen y distribución de los incendios forestales en el estado de México (2000 a 2011). Revista Mexicana de Ciencias Forestales 6(29):92-107.

Middleton, BA; Sanchez-Rojas, E; Suedmeyer, B; Michels, A. 1997. Fire in a tropical dry forest of Central America: a natural part of the disturbance regime?. Biotropica 29(4):515-517.

MINAGRI (Ministerio de Agricultura y Riego, Perú). 2016. Evolución de producción del sector forestal: noviembre 2016 (en línea). Consultado 27 dic. 2020. Disponible en http://www. peruforestal.org/estadisticas/.

MINAM (Ministerio del Ambiente, Perú). 2019. Mapa nacional de ecosistemas: memoria descriptiva (en línea). Consultado 15 dic. 2020. Disponible en https://geoservidor.minam.gob. pe/wp-content/uploads/2019/11/Memoria Mapa Ecosistemas Peru.pdf.

Moscovich, FA; Dummel, C; Pinazo, MA; Knebel, O; Alcaraz, R. 2010. Fitosociología de una fracción de selva misionera secundaria, con intervención antrópica. In Jornadas Técnicas 
Forestales y Ambientales (14, 2010, Misiones, Argentina). Misiones, Argentina, UNAM.

Numata, I; Silva, SS; Cochrane, MA; d'Oliveira, MV. 2017. Fire and edge effects in a fragmented tropical forest landscape in the southwestern Amazon. Forest Ecology and Management (401):135-146.

Oksanen, J; Blanchet, F; Friendly, M; Kindt, R; Legendre, P; McGlinn, D; Minchin, P; O’ Hara, RB; Simpson, GL; Solymos, P; Stevens, MH; Szoecs, E; Wagner, H. 2019. Vegan: Community Ecology Package (en línea). R package vers. 2.5-6. Consultado 15 dic. 2020. Disponible en https://CRAN.R-project.org/package=vegan.

Otterstrom, SM; Schwartz, MW; Velázquez-Rocha, I. 2006. Responses to fire in selected tropical dry forest trees. Biotropica 38(5):592-598.

Palacios, S. 2017. Dinámica forestal de una formación vegetal subxerófila en el valle de Chanchamayo, Dpto. Junín. Tesis Mg Sc. Lima, Perú, UNALM. 157 p.

Palacios, S; Montenegro, R; Linares-Palomino, R; Reynel, C. 2018. Forest dynamics of a sub-xerophilous vegetation formation in central Peru-Chanchamayo valley, Peru. Revista Árvore 42(6):e420603.

Palacios, S; Reynel, C. 2011. Una formación vegetal subxerófila en el valle de Chanchamayo, Dp. Junín (Perú). Lima, Perú, UNALM. 72 p.

Pennington, RT; Lehmann, CER; Rowland, LM. 2018. Tropical Savannas and dry forests. Current Biology 28(9):R541-R545.

Pereira, IS; Calil, FN; Martins, TO; Silva-Neto, CDM; Borges, JB; Venturoli, F; Oliveira, LH. 2017. Fire effect on the seasonal forest structure in the Cerrado biome. Floresta 46(4):499507.

Quintero, F. 2019.Características de la sucesión vegetal en el valle de Chanchamayo y sus implicancias para su conservación y manejo forestal. Tesis Mg Sc. Lima, Perú, UNALM. 360 p.

Quintero Cardozo, F.; Cáceres Rodríguez, BM.; Reynel Rodríguez, CA.; Fernandez-Hilario, R.; Wong Sato, AA; Chávez Salas, JM.; Palacios Ra- mos, SC. 2020. Tiempos de recomposición de la diversidad arbórea a lo largo de la sucesión vegetal en los bosques del valle de Chanchamayo / Junín / Perú. Ecología aplicada 19(2):111-120.

R Core Team. 2020. R: A language and environment for statistical computing (en línea). Vienna, Austria, R Foundation for Statistical Computing. Consultado 15 dic. 2020. Disponible en https://www.R-project.org/.

Sardi, A; Torres, AM; Corredor, G. 2018. Diversidad florística en un paisaje rural del piedemonte de los Farallones de Cali, Colombia. Colombia forestal 21(2):142-160.

SENAMHI (Servicio Nacional de Meteorología e Hidrología del Perú). 2018. Mapa de clasificación climática del Perú (en línea). Lima, Perú. 9 p. Consultado 16 dic. 2020. Disponible en http://idesep.senamhi.gob.pe/geonetwork/srv/ spa/catalog.search\#/metadata/d4518248-af564419-9b5d-b42de7dba471.

SERFOR (Servicio Nacional Forestal y de Fauna Silvestre, Perú). 2018. Plan de prevención y reducción de riesgos de incendios forestales. Lima, Perú. Consultado 10 jun. 2021. Disponible en https://www.serfor.gob.pe/portal/ wp-content/uploads/2018/12/Plan-de-prevenci\%C3\%B3n-y-reducci\%C3\%B3n-de-riesgos-de-incendios-forestales.pdf.

Simon, MF; Pennington, T. 2012. Evidence for adaptation to fire regimes in the tropical savannas of the brazilian Cerrado. International Journal of Plant Sciences 173(6):711-723.

Silveira, AO; Fernandes, GW; Lemos-Filho, JP. 2013. Seed and seedling ecophysiology of Neotropical Melastomataceae: implications for conservation and restoration of savannas and rainforests. Annals of the Missouri Botanical Garden 99(1):82-99.

Vásquez, R; Rojas, R. 2016. Clave para identificar grupos de familias de Gymnospermae y Angiospermae del Perú. Lima, Perú, Jardín Botánico de Missouri.79 p.

Vásquez, R; Rojas, R; Monteagudo, A; Valenzuela, L; Huamantupa, I. 2018. Catálogo de árboles del Perú. Q’EUÑA 9(1):1-607. 
Composición florística y diversidad arbórea post quema de

Xavier, KRF; de Andrade, LA; Fabricante, JR; Coelho, MDSE; de Medeiros Assis, FN. 2011. Impactos pós-fogo na regeneração natural em um fragmento de floresta ombrófila aberta no município de Areia, Paraíba, Brasil. Revista brasileira de Biociências 9(3):257-264. 